\title{
Direct interaction of Gas41 and Myc encoded by amplified genes in nervous system tumours
}

\author{
Eugenia Piccinni ${ }^{1}$, Anna Chelstowska ${ }^{2}$, Jakub Hanus ${ }^{3}$, Piotr Widlak ${ }^{3}$, Simona Loreti ${ }^{1}$, \\ Ada Maria Tata', Gabriella Augusti-Tocco ${ }^{1}$, Michele Maria Bianchi ${ }^{1 凶}$ and Rodolfo Negri ${ }^{\bowtie}$
}

1Dipartimento di Biologia e Biotecnologie 'Charles Darwin' and Istituto Pasteur-Fondazione Cenci Bolognetti, Sapienza Università di Roma, Roma Italy; ${ }^{2}$ Department of Genetics, Institute of Biochemistry and Biophysics, Polish Academy of Sciences, Warsaw, Poland; ${ }^{3}$ Maria Sklodowska-Curie Memorial Cancer Center and Institute of Oncology, Gliwice, Poland

In order to understand better the role of the human Tip60 complex component Gas41, we analysed its expression levels in brain tumours and searched for possible interactors. Two-hybrid screening of a human foetal brain library allowed identification of some molecular interactors of Gas41. Among them we found nMyc transcription factor. The interaction between Gas41 and $n$-Myc was validated by pull-down experiments. We showed that Gas41 is able to bind both n-Myc and c-Myc proteins, and that the levels of expression of Gas41 and Myc proteins were similar to each other in such brain tumors as neuroblastomas and glioblastomas. Finally, in order to identify which region of Gas41 is involved in the interaction with Myc proteins, we analysed the ability of Gas41 to substitute for its orthologue Yaf9 in yeast; we showed that the N-terminal portions of the two proteins, containing the YEATS domains, are interchangeable, while the C-terminal portions are species-specific. In fact we found that Gas41 C-terminal portion is required for Myc protein interaction in human.

Keywords: Gas41, n-Myc, transcription regulation, chromatin modification, brain tumours

Received: 21 March, 2011; revised: 14 September, 2011; accepted: 13 October, 2011; available on-line: 08 November, 2011

\section{INTRODUCTION}

The glioma-amplified sequence (gas) 41 gene was identified for the first time as an amplified sequence in the human chromosome region 12q13-15, a locus known to be involved in gene amplification in human gliomas. Genomic amplifications are frequently found in human tumours and thought to be an indication of the intrinsic genome instability of the cancer cells. This gene was found to be amplified in $23 \%$ of glioblastomas and in $80 \%$ of grade I astrocytomas, suggesting that gene amplification occurs also in early stages of cancerogenesis (Fischer et al., 1996; 1997; Zimmermann et al., 2002). Gas41 is a highly conserved protein (Harborth et al., 2000; Zimmermann et al., 2002; Le Masson et al., 2003). Sequence comparison indicates high homology of Gas41 with the human transcription factors AF-9 and ENL. Differently from ENL and AF-9, Gas41 does not have a typical DNA binding domain, suggesting a mediated interaction with DNA targets. Gas41 has a nuclear localization and interacts with many complexes and proteins: the nuclear mitotic apparatus (NuMa), the prefoldinlike protein subunit 1 (PFDN1), the KIAA1009 protein and the MLL-AF10 fusion protein, which is detected in $5-10 \%$ of human acute leukaemias (Debernardi et al., 2002).

Particularly informative for the function of gas 41 are studies on its yeast homologous gene YAF9 (Yeast AF9). YAF9 encodes a 226-aminoacid protein with $53 \%$ identity and $80 \%$ similarity with Gas41. Most of the homology is in the N-terminal part (Fischer et al., 1997; Le Masson et al., 2003). YAF9 is a non-essential yeast gene but the yaf9s strain shows various growth phenotypes (Bianchi et al., 2001). Yaf9 is involved in transcriptional regulation of several genes (Le Masson et al., 2003; Bianchi et al., 2004; Del Vescovo et al., 2008; Casagrande et al., 2009) and binds to regulated promoters, affecting histone acetylation levels (Bianchi et al., 2004). Yaf9 is present in the NuA4 histone acetyltransferase complex (Bianchi et al., 2004) and in the SWR1-complex (SWR1-C) involved in the substitution of histone H2A with H2AZ (Krogan et al., 2003; Mizuguchi et al., 2004; Zhang et al., 2005).

In human, Gas41 is a common subunit of the SRCAP and Tip60 complexes which are the mammalian version of SWR1 and NuA4 complexes, respectively; also in Drosophila, Gas41 is involved in a complex which appears to correspond to NuA4 and SWR1 yeast complexes (Doyon et al., 2004; Doyon \& Cote 2004; Schultze et al., 2009). These complexes change chromatin structure and allow gene transcription, suggesting that Yaf9 and Gas41 might promote the recruitment of these complexes to regulated promoters in yeast and mammalian cells, respectively. Interestingly, Yaf9 seems be required also for the cellular response to spindle stress in yeast, a function correlated to the recruitment of NuA4 to the centromeric chromatin (Le Masson et al., 2003; Krogan et al., 2003). On the other hand, Gas41 has been shown to interact with INI (Integrase Interactor 1) which is the human homologue of the yeast SNF5, a component of the SWI/SNF chromatin remodelling complex (Debernardi et al., 2002), and with TACC1 and TACC2 proteins (Gangisetty et al., 2004). TACC proteins are involved in the control of cell growth and differentiation and might be implicated in the development of multiple myeloma, breast and gastric cancer. These proteins interact with the centrosome and with INI probably through their interaction with Gas41.

E-mail: rodolfo.negri@uniroma1.it; michele.bianchi@uniroma1.it Abbreviations: DAPI: 4,6-diamidino-2-phenyl indole, DMSO: dimethyl sulfoxide, EGTA: ethylene glycol tetraacetic acid, PMSF: phenyl methane sulfonyl fluoride. 
However, in spite of these observations, it is still unclear to what extent the function of these proteins is evolutionarily conserved and whether Gas41 retains in mammals the role that Yaf9 seems to have in yeast. If this is the case, yeast would be further confirmed as an interesting model to investigate Gas41 role in the emergence of brain tumours and possibly their degree of malignancy.

In the present work we searched for possible interactors for Gas41 and demonstrated that it can interact with n-Myc and c-Myc proteins; the possible relationship between Gas41 and n-Myc expression was investigated in brain tumours. Finally, we proved that in yeast Gas41 $\mathrm{N}$-terminal portion can functionally substitute for its Yaf9 counterpart.

\section{MATERIALS AND METHODS}

Peptides and antibodies. Recombinant Gas41 protein T7-tagged at N-terminus was from Abcam (Cambridge, UK: ab40052). Recombinant c-Myc (64 kDa) was from Active Motif (Rixensart, BE: 31117). Synthetic $\mathrm{n}$-Myc peptide derived from residues 1-100 of human n-Myc was from Abcam (ab31595). Primary Antibodies: c-Myc (c33) mouse monoclonal antibody for detection of c-Myc p67 was from Santa Cruz Biotechnology (Santa Cruz, CA, USA). Mouse monoclonal to n-Myc (ab16898), chicken polyclonal to Gas41 (ab15859) and goat polyclonal anti-T7 tag-agarose (ab1230) were from Abcam.

Cell cultures. Human neuroblastoma cell lines SK-NSH and IMR32 (ATCC) and normal human fibroblasts MRC-5 (ATCC) were cultured in Minimum Essential Medium (MEM) and Dulbecco's modified Minimum Essential Medium (DMEM) supplemented with 1\% Non Essential Amino Acids (NEAA), 1\% glutamine (200 $\mathrm{mM})$, sodium pyruvate $(110 \mathrm{mg} / \mathrm{ml}), 10 \%$ fetal calf serum (FCS) (Sigma), penicillin $(5000 \mathrm{IU} / \mathrm{ml}$ ) and streptomycin $(5 \mathrm{mg} / \mathrm{ml})$.

Cell extract preparation. Whole cell extracts, cytoplasmic fractions and nuclear fractions were prepared with Nuclear Extract Kit (Active Motif, Carlsbad, CA, USA: 40010) according to the manufacturer's instructions. The protein content of the fractions was quantified by Bradford method (Bradford 1976).

Construction of hybrid proteins. The gas 41 coding sequence was amplified by PCR from pBKCMVgas41 (kind gift of U. Fischer) and cloned into vector pUG35. The primers used (Table 1) introduced a BamHI site at the 5' end (G5B) and an in-frame SalI site at the 3' end (G3S). The resulting vector contained a gas $41::$ GFP fusion gene regulated by the MET25 promoter. The following gene sequences were amplified with the indicated pairs of primers: gas $41=2 \mathrm{HP} 1$ and 2HP2; gas41 N-half = 2HP1 and 2HP3; gas41 C-half $=2 \mathrm{HP} 4$ and $2 \mathrm{HP} 2 ; \mathrm{YAF} 9=2 \mathrm{HP} 5$ and $2 \mathrm{HP} 6$; YAF9 $\mathrm{N}$-half $=2 \mathrm{HP} 5$ and $2 \mathrm{HP} 7 ;$ Y AF9 $\mathrm{C}$-half $=2 \mathrm{HP} 8$ and 2HP6. The oligonucleotides used as primers introduced SmaI (2HP1, 2HP5), PstI (2HP2, 2HPG) or SalI (2HP3, 2HP4, $2 \mathrm{HP} 7$ and 2HP8) sites. The fragments amplified were cut with the corresponding enzymes and inserted into pGBKT7 plasmid (Bittner et al., 2004). The hybrid protein composed of the N-terminal half of Gas41 fused to the C-terminal half of Yaf9 was also cloned in the pESC-URA vector. To this purpose, the oligonucleotides used as primers introduced BamHI (GY5B) and AvaI sites (GY3A).
Table 1. Primer list

\begin{tabular}{ll}
\hline Name & Sequence \\
\hline G5B & CGGGATCCATGTTCAAGAGAATGGCCGA \\
G3S & ACGCGTCGACTATGTCTITGCTTGGTC \\
2HP1 & TCCCCCGGGGATGTTCAAGAGAATGGCCGA \\
2HP2 & AAACTGCAGTTATATGTCTITGCTTGG \\
2HP3 & CGCGTCGACAAACAGCTTAGCAAATGA \\
2HP4 & CGCGTCGACCAATCAGACACCAATGCAA \\
2HP5 & TCCCCCGGGGATGGCTCCGACAATAAGCA \\
2HP6 & AAACTGCAGCTAACTTCCGTTAATGGC \\
2HP7 & CGCGTCGACAGCATAAGGATGAAGTCG \\
2HP8 & CGCGTCGACGCTAATCCTGTACCGAAT \\
GY5B & CGCGGATCCATGTTCAAGAGAATGGCCG \\
GY3A & TCCCTCGAGACTTCCGTTAATGGCTTC \\
\hline
\end{tabular}

Yeast two-hybrid analysis. The Matchmaker yeast two-hybrid system and the pre-transformed human foetal brain cDNA library were obtained from Clontech Laboratories (Palo Alto, CA, USA). After constructing pGBKT7-GAS41, the bait plasmid was transformed into yeast strain AH109 using the lithium acetate method and checked for absence of autoactivation or non-specific interactions between Gas41 and the GAL4 activation domain. Diploids that activated the HIS3, ADE2 and $L a c Z$ reporter genes only in the presence of the pGBKT7-gas41 plasmid were considered positive. The pACT2 plasmids containing cDNAs encoding potential Gas41 interacting proteins were isolated as suggested by the supplier (Clontech Laboratories; Yeast Protocols Handbook PT3024-1), transformed into Escherichia coli cells (strain DH5-a; Invitrogen, Carlsbad, CA, USA) and sequenced.

Coupling of Gas41 agarose beads. Eight $\mu$ l of recombinant T7-tagged Gas41 protein $(0.5 \mu \mathrm{g} / \mu \mathrm{l})$ was combined with $30 \mu \mathrm{l}$ of anti-T7 tag agarose beads previously equilibrated in buffer $\mathrm{G}(20 \mathrm{mM}$ Hepes $\mathrm{pH}$ 7.5, $100 \mathrm{mM} \mathrm{NaCl}, 10 \%$ glycerol, $1.5 \mathrm{mM} \mathrm{MgCl}_{2}, 1$ $\mathrm{mM} \mathrm{CaCl}, 1 \mathrm{mM} \mathrm{EGTA,} \mathrm{protease} \mathrm{inhibitors} \mathrm{cocktail).}$ The mixture was kept at $4^{\circ} \mathrm{C}$ with mild agitation for 3 h. The beads were subsequently pelleted at $2000 \mathrm{~g}$ and the supernatant removed (FT, flow through). The beads were washed 4 times with 1 volume of buffer $G$ and each time pelleted at $2000 \mathrm{~g}$. No protein leakage was observed and the pelleted beads were immediately used for pull-down experiments.

Pull-down with recombinant c-Myc or n-Myc. Thirty $\mu$ l of anti-T7 tag beads coupled with T7-tagged Gas41 was mixed with $30 \mu \mathrm{l}$ of buffer $\mathrm{G}$ containing recombinant c-Myc or $\mathrm{n}-\mathrm{Myc}$ peptides (ranging from 4 to $10 \mu \mathrm{g}$ ) and kept for $20 \mathrm{~min}$ at $4^{\circ} \mathrm{C}$ with mild agitation. The beads were subsequently pelleted and the supernatant removed (FT). The beads were washed 4 times and supernatants stored (W1-W4). The bound protein was eluted with $30 \mu \mathrm{l}$ of sample buffer $(62.5 \mathrm{mM}$ Tris/HCL, $\mathrm{pH} 6.8,2 \%$ SDS, $10 \%$ glycerol). As a control the same experiment was performed with $30 \mu \mathrm{l}$ of anti-T7 tag-agarose not coupled to Gas41.

Pull-down cellular fractions. Thirty $\mu \mathrm{g}$ of IMR32 nuclear or cytoplasmic fraction, prepared as described, was mixed with $30 \mu \mathrm{l}$ of anti-T7 tag beads coupled with T7-tagged Gas41. The beads were subsequently pelleted and the supernatant removed (FT). The beads were 


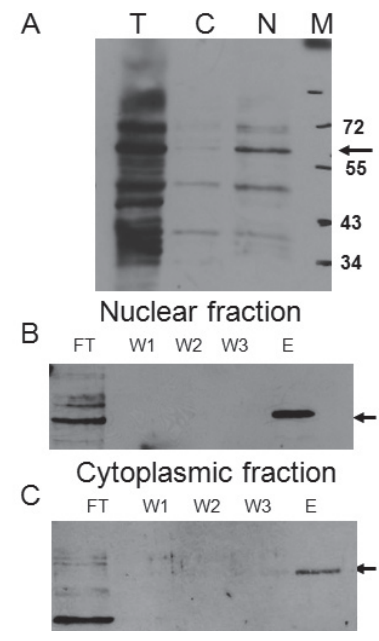

Figure 1. Pull-down experiments confirm Gas41-n-Myc interaction.

(A) Western blot of IMR32 total cell extract (T); cytoplasmic (C) and nuclear (F) fractions, and marker (M). (B and C) samples of supernatant of flow through (FT); wash (W1-W3) and elution (E) of pull down experiments performed with nuclear (B) or cytoplasmic (C) fractions on beads coupled to Gas41. Fractions (half volume each) were analysed by western blot. The arrows indicate unphosphorylated n-Myc (64 kDa).

washed 3 times and supernatants stored (W1-W3). The bound proteins were eluted with $30 \mu$ l of sample buffer. As a control the same experiment was performed with $30 \mu \mathrm{l}$ of anti-T7 tag beads without Gas41.

Western blotting. Total proteins were extracted from tumour cell lines by RIPA buffer $(50 \mathrm{mM}$ Tris- $\mathrm{HCl} \mathrm{pH}$ 7.4, $150 \mathrm{mM} \mathrm{NaCl}, 1 \%$ NP40, $1 \mathrm{mM}$ PMSF, 1x protease inhibitor cocktail, Sigma), run in $12 \%$ SDS-PAGE and blotted on nitrocellulose membrane ProteanBA85 (Schleicher \& Schuell/Whatman, Maidstone, Kent, UK). Membranes were incubated with anti-Gas41, anti-c-Myc or anti-n-Myc antibodies. The secondary HRP-conjugated antibodies used for the detection were rabbit polyclonal anti-chicken $\operatorname{IgY}$ (Abcam) and rabbit polyclonal antimouse IgG (Abcam), respectively. The enzymatic activity was revealed using ECL-detection kit (Pierce Biotechnology, Rockford, IL, USA). Anti-actin antibody (Chemicon/ Millipore, Temecula, CA, USA) was used as reference.

gas 41 and $\mathrm{n}-\boldsymbol{m y c}$ expression. Total RNA isolated from biopsies of 13 human brain tumours ( 5 glioblastomas, 4 anaplastic astrocytomas, 4 tumours with unclear histology) were analyzed using the AFFY_HG_U133 PLUS_2 Affymetrix microarrays. Expression levels of gas41 (218911_at probeset) and n-myc (209756_s_at and 211377_x_at probesets) genes were calculated from complete raw data set after a standard GC-RMA pre-processing. The correlation analysis was performed with average values extracted from two independent experiments.

\section{RESULTS}

\section{Identification of Gas41 interactors}

The function of Gas41 could be clarified by the identification of physically interacting proteins. We therefore performed screening of a human foetal-brain cDNA library in yeast with the two-hybrid method, using Gas41 as bait. We constructed the Gas41 bait fused with the GAL4 DNA-BD and we transformed a suitable mating partner (strain AH109, MAT a). The human foetal
Table 2. Gas41 interactors

\begin{tabular}{lll}
\hline Protein & Number of isolates & Interaction with Yaf9 ${ }^{1}$ \\
\hline n-Myc & 6 & Clone dependent ${ }^{2}$ \\
Lsd1 & 2 & - \\
FRMD4A & 2 & + \\
Rnf8 & 1 & +++ \\
Hs Chr 17 ORF70 & 1 & Not tested \\
\hline
\end{tabular}

${ }^{1}+++=$ strong interaction; $+=$ weak interaction; $-=$ not interacting; 2from strong to weak.

brain cDNA library, expressing proteins fused with the GAL4-AD, was provided in the yeast strain Y187 (MAT $\alpha)$. The two transformed cultures were mated to each other and diploid cells were selected. By plating the diploids on selective medium, only those containing putative interacting proteins could express the selectable marker gene(s) and grow. About $3 \times 10^{6}$ diploid clones were analysed in two independent screenings. Positive clones were tested also for interaction with Yaf9 (Table 2). Six proteins were confirmed as true interactors of Gas41: the cytoskeletal protein FRMD4A (two isolates) and the ubiquitinationrelated protein Rnf8 (one isolate), which both interacted also with Yaf9. Six isolates contained the oncogenic transcription factor n-Myc and interaction with Yaf9 was clone-dependent, probably because some of the isolates contained incomplete cDNA. Lsd1 demethylase (two isolates), a protein involved in chromatin modification, did not interact with Yaf9. One isolate contained ORF70 (HS chr17), whose interaction with Yaf9 was not tested.

\section{Validation of Gas41/n-Myc interaction in human cell lines}

Among the identified Gas41 interactors we decided to further analyze Myc, because of its well known role in cell proliferation and its amplification in tumours, e.g., neuroblastomas. IMR32 neuroblastoma cell line showed
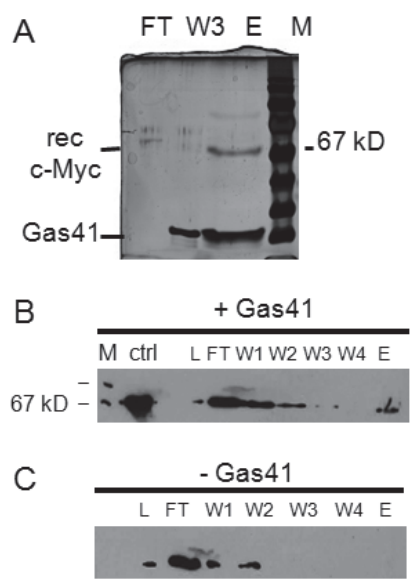

Figure 2. Purified recombinant c-Myc interacts with Gas41 in vitro.

(A) Silver stained protein gel (12\% SDS/PAGE) showing a sample of supernatant of indicated fractions (FT: flow through; W3: third wash; E: eluate) of a pull-down experiment performed with recombinant c-Myc on beads coupled to Gas41. Half volume of each fraction was analysed. Position of Gas41 and recombinant c-Myc is indicated. (B) Western blot of a protein gel carrying indicated fractions (L: loaded sample; FT: flow through; W1-W4: washes; E: eluate) of a pull-down experiment performed with recombinant $c^{-}$ Myc on beads coupled to Gas41. 1/50 th of volume of the load and $1 / 20^{\text {th }}$ of each fraction were analysed. $M=M W$ marker, $c$ trl=c-Myc. (C) Same experiment as in Fig. 3B, but performed with uncoupled beads. 


\section{A}

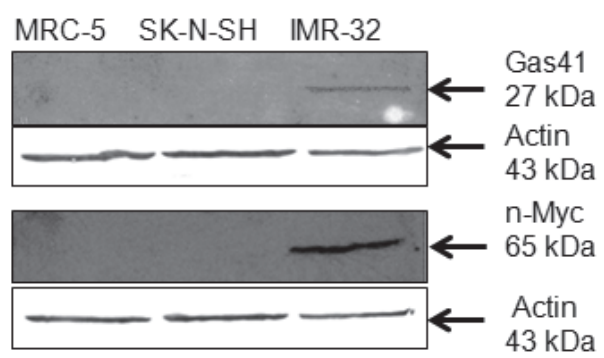

B

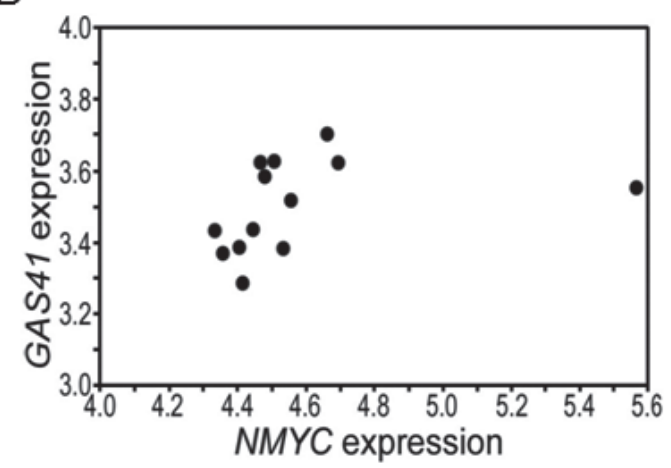

Figure 3. Gas41 and n-Myc show similar expression levels in brain tumours.

(A) Western blot analysis of MRC-5, SK-N-SH and IMR32 protein lysates. Arrows indicate Gas41 (27 kDa), n-Myc (64 kDa) and actin (43 kDa) signals. (B) Expression levels of gas 41 and $\mathrm{n}$-myc genes were extracted from complete microrray analysis (Affymetrix) of tissue biopsies from 13 human brain tumours. Each dot represents expression levels for each tumour sample ( $\log _{2}$ expression signals).

a very high expression level of n-Myc (Schwab et al., 1984) and therefore appeared an ideal system for validating its interaction with Gas41 by biochemical assays. Cytoplasmic and nuclear fractions were used for pull-down experiments with agarose beads coupled with Gas41. Figure $1 \mathrm{~A}$ shows that both total cell extract and nuclear fraction contained a major n-Myc band and smaller (probably proteolytic) fragments. The nuclear extract showed two additional larger bands which could be nuclear phosphorylated forms. Both in the nuclear and in

Table 3. Yaf9 and Gas41 vectors.

\begin{tabular}{llllll}
\hline \multirow{2}{*}{ Protein $^{1}$} & \multirow{2}{*}{ Vector $^{2}$} & Promoter & \multicolumn{3}{l}{ Phenotypes $^{3}$} \\
\cline { 5 - 6 } & & & DMSO & $\mathrm{CsCl}$ & Benomyl \\
\hline Yaf9 (entire) & pUG35 (GFP) & MET25 & + & + & + \\
Gas41 (entire) & " & " & - & - & - \\
Yaf9 (entire) & pGBT9 (DNA-BD) & trADH14 & + & - & - \\
Gas41 (N)+Yaf9 (C) & $"$ & " & + & - & - \\
Yaf9 (N)+GAS41 (C) & $"$ & " & - & - & - \\
Gas41 (entire) & $"$ & " & - & - & - \\
Gas41 (N)+Yaf9 (C) & pESC-URA & GAL1 & + & + & + \\
Yaf9 (entire) & pGBKT7 (DNA-BD, c-myc) & ADH1 & + & + & + \\
Yaf9 (N) only & $"$ & " & - & - & - \\
Yaf9 (C) only & " & " & - & - & - \\
\hline
\end{tabular}

$1 \mathrm{~N}$-half (N) and C-half (C) of Yaf9 protein were aminoacids 1 to 121 and 116 to 226 , respectively. $\mathrm{N}$-half (N) and C-half (C) of Gas41 were 1 to 121 and 122 to 227 , respectively; ${ }^{2}$ In parentheses the genetic elements fused to the gene sequence are indicated; ${ }^{3}+$ and - indicate growth and non-growth on the indicated xenobiotic; ${ }^{4} \mathrm{tr} A D H 1$ is a truncated form of the $A D H 1$ promoter with reduced transcription activity; ${ }^{5}$ This set of vectors was kindly provided by R. Slany (Bittner et al., 2004). the cytoplasmic fraction (Fig. 1B and 1C), n-Myc seemed to bind to the Gas41-coupled beads mainly in the unphosphorylated form. No binding was observed with beads not coupled to Gas41 (not shown).

Since c-Myc can interact with the Tip60 complex through the Trrap protein component (McMahon et al., 2000), we could not exclude that Trrap present in the extracts might mediate $\mathrm{n}-\mathrm{Myc}$ binding to Gas41. To rule out this hypothesis we performed pull-down experiments using a purified recombinant c-Myc. We could detect c-Myc binding both by silver staining (Fig. 2A) and by western blotting with an antibody specifically recognizing c-Myc (Fig. 2B). When performing the pull-down with beads uncoupled to Gas41 (Fig. 2C), we did not detect c-Myc binding. This experiment demonstrates a direct interaction between c-Myc and Gas41. Since c-Myc and n-Myc share extensive homology at the C-terminal part, it is likely that interaction of both proteins with Gas41 is mediated by this portion. This hypothesis was confirmed by the absence of binding when pull-down was performed with 100 aminoacids of the N-terminal portion of n-Myc (synthetic peptide; data not shown).

\section{Gas41 and n-Myc expression in brain tumours}

We tested whether n-Myc and Gas41 were expressed at comparable levels in tumour cell lines. We analysed Gas41 and n-Myc expression in human neuroblastoma cell lines SK-N-SH and IMR32. In SK-N-SH cells, n-myc is not significantly amplified and/or expressed (Schwab et al., 1984). It has been estimated that in IMR32 cells $\mathrm{n}$-myc is amplified 15-20-fold and has 60-80-fold enhanced protein expression, as compared with SK-N-SH. We performed western blotting on protein extracts from cell lines SK-N-SH and IMR32, using anti-n-Myc monoclonal antibody and anti-Gas41 polyclonal antibody to detect protein levels. n-Myc and Gas41 signals appeared only in IMR32 neuroblastoma cell line (Fig. 3A). Moreover, $\mathrm{n}-m y c$ RNA is $5-20$-fold higher in IMR32 than in SK-N-SH (not shown). These results suggested that the over-expression of gas 41 and n-myc could be correlated events. In order to support this hypothesis we performed trascriptome analysis of biopsies from 13 human brain tumours (5 glioblastomas, 4 anaplastic astrocytomas, 4 tumours with unclear histology). A positive correlation $(\mathrm{r}=0.31, \quad p$-value $=0.001)$ between the expression level of the two proteins was found (Fig. 3B). In order to test if there was a direct effect of Gas41 over-expression on nMyc over-expression or vice versa, we transfected NIHH1299 cells with FLAG-tagged Gas41 or with FLAG-tagged $\mathrm{n}-\mathrm{Myc}$ and analyzed protein and RNA levels. No clear effect was observed, excluding a direct reciprocal regulation (not shown).

\section{Expression of Gas41 in S. cerevisiae}

Gas41 and Yaf9 share significant sequence homology (Le Masson et al., 2003) but 
A

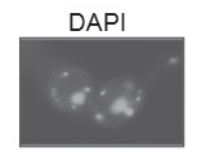

$B$ DMSO $3 \%$

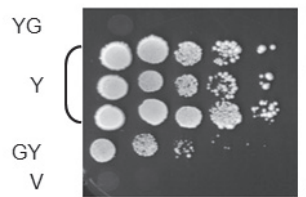

C YPGal $2 \%$ Glu $0,5 \%$

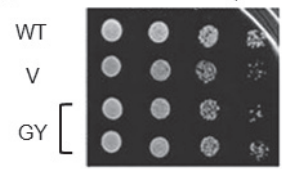

DMSO $3 \%$
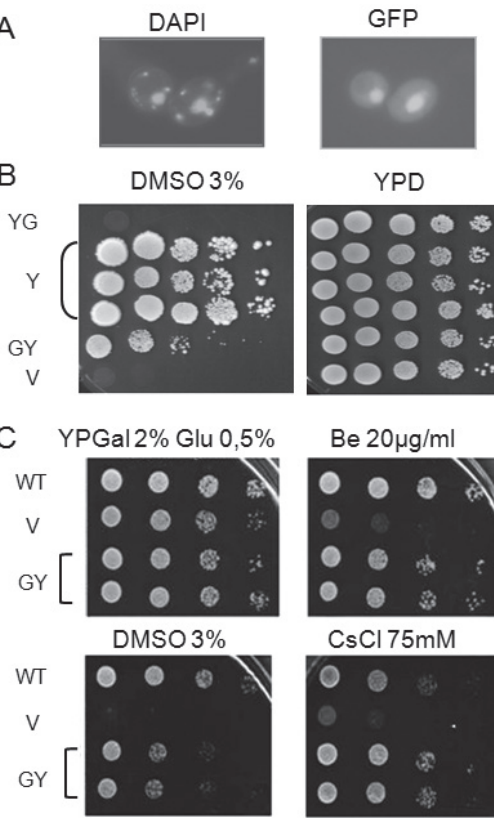

YPD

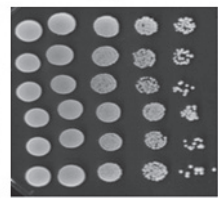

$\mathrm{Be} 20 \mu \mathrm{g} / \mathrm{ml}$

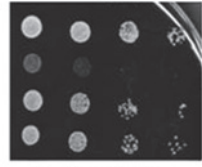

$\mathrm{CsCl} 75 \mathrm{mM}$

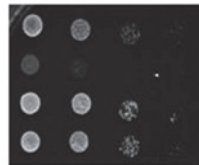

Figure 4. Gas41 and Yaf9 amino-terminal portions are interchangeable.

(A) Nuclear localization in yeast of human Gas41::GFP protein (right). Cell stained with DAPI (left) shows nuclei positioning. (B) Phenotypic suppression of DMSO sensitivity (left) of yaf9 $\Delta$ strain transformed with hybrid proteins. Gas41 N-half and Yaf9 C-half: row GY. Reciprocal hybrid protein: row YG. Positive (three independent clones) and negative controls: rows $\mathrm{Y}$ (entire Yaf9) and $\mathrm{V}$ (vector), respectively. Growth on medium (YPD) without inhibitor is shown at right. (C) Suppression by hybrid protein (two clones, rows GY) of sensitivity to Benomyl (upper-right), DMSO (lower-left) and $\mathrm{CsCl}$ (lower-right) of yaf9 $\Delta$ strain. Controls are wild-type (row WT) strain and yafg $\Delta$ strain transformed with the empty vector (V). Growth on medium (YPGal-Glu) without inhibitors is reported in the upper-left.

it is still unclear to what extent they can be considered functional homologues. We assayed a functional substitution of Yaf9 with Gas41 in yeast by cloning gas41 cDNA, kindly provided by U. Fischer, into the centromeric yeast vector pUG35 and generating a fusion protein with GFP. After transformation of the yaf9s strain, we observed by fluorescent microscopy that Gas41 was expressed and was localized in the nucleus (Fig. 4A), similarly to what was previously observed with a Yaf9GFP fusion (Le Masson et al., 2003). The yaf9s strain is sensitive to $\mathrm{CsCl}, \mathrm{DMSO}$ and Benomyl (Bianchi et al., 2004): however, the expression of Gas41 did not suppress any of the yaf9s strain phenotypes (Table 3) suggesting that Gas41 was lacking functionality of domain(s) required for biological functions in yeast. To identify these domains, we constructed Gas41-Yaf9 hybrid proteins having exchanged $\mathrm{N}$-halves and C-halves, as described in Table 3, and we tested them for phenotype suppression. The chimeric genes were cloned in vectors pGBT9 and pESC-URA and used to transform yaf9s strain. The presence of the expected proteins in the transformed clones was verified by western blotting (not shown). Results of suppression (Fig. 4B and 4C) clearly showed that only the chimeric protein composed of the N-terminal half of Gas41 and the C-terminal half of Yaf9 was able to restore growth on inhibitors. Results also suggested that the level of gene expression, i.e. strength of the promoter, might specifically influence the resistance to individual inhibitors. Control experiments showed that expression of only the $\mathrm{N}$-terminal half or the C-terminal half of Yaf9 was unable to suppress sen- sitivity to inhibitors (Table 3). These findings indicated that the N-terminal halves of Gas41 and Yaf9, whose protein sequences are more conserved than the C-terminal halves (Le Masson et al., 2003), were functionally exchangeable. The inability of the C-terminal half of Gas41 to restore growth and the sequence divergence of Gas41 and Yaf9 in these portions suggested that species specific functions might rely on the C-terminal half.

\section{DISCUSSION}

We have identified six new Gas41 molecular interactors, including two proteins involved in regulation of gene expression: Lsd1 and n-Myc. We focused our attention to the latter and performed experiments to confirm the interaction between Gas41 and n-Myc, while the interactions between Gas41 and the other five proteins will require further in vivo and/or in vitro investigations. n-Myc is a member of a family of oncoproteins involved in transcription regulation and targeting of chromatin modification complexes at active promoters (Patel et al., 2004; Martinato et al., 2008; Perini et al., 2005). In our two-hybrid screening with Gas41, we isolated six clones containing different $\mathrm{n}$-Myc portions. Interaction with Yaf9 was clone-dependent, suggesting that different domains of $\mathrm{n}$ Myc could be involved in the interaction; however, in vitro binding of Gas41 with the N-terminal part of n-Myc did not occur. Our results indicate that also c-Myc can directly interact with Gas41. It has been shown that the HATcontaining Tip60 complex is targeted to active promoters by c-Myc through an interaction with the Trrap subunit (McMahon et al., 2000; Martinato et al., 2008; Frank et al., 2003) involving the N-terminal portion of c-Myc (McMahon et al., 2000). Since c-Myc and n-Myc share extensive homology at the C-terminus, our results suggest a second targeting mode, mediated by Gas41, which could be used by Myc proteins to recruit chromatin modification machineries. The interaction between Myc proteins and Gas41 might also be confirmed by additional approaches, such as reverse pull-down experiments.

In addition to their physical interaction, a correlation between Gas41 and $\mathrm{n}-\mathrm{Myc}$ is also suggested by their overexpression in tumour cell line IMR32 and in several brain tumours, independently of malignancy degree. However, although the P-value associated with the co-expression of Gas41 and $\mathrm{n}-\mathrm{Myc}$ is low $(P<0.001)$, the detail of the functional interaction between the two proteins remains to be demonstrated. Moreover, it does not seem to derive from a direct reciprocal regulatory control of the two genes, as suggested by experiments in NIH-H1299 cells. We can only speculate that n-myc amplification in brain tumours might select gas41 amplifying or over-expressing cells which can better exploit the augmented targeting capability of n-Myc. The crucial importance of this recruiting activity for transcription regulation is probably at the base of the evolution of multiple interactions between the Myc proteins and Tip60 and SCRAP complex subunits. It has been proposed that Gas41 (Doyon \& Cote 2004), as its yeast counterpart Yaf9, is associated with other three subunits (Dmap1, Baf53 and actin) in a recruiting module distinct from the one containing Trrap. It is possible that the two different modules have different interaction partners and different influence on the targeting of Tip60 and SCRAP.

Lsd1 is a FAD-dependent amine oxidase which promotes histone demethylation and which has been found in complexes commonly involved in repression of transcription. However, Lsd1 has been found to operate also 
in transcription activation (Metzer \& Schule 2007; Perillo et al., 2008) and a model of cooperation between Lsd1 and $\mathrm{Myc}$ has been proposed for gene expression (Amente et al., 2010). Although not yet demonstrated in human cells, we have found that Lsd1 specifically interacts with Gas41 in yeast, suggesting that the cooperation between Lsd1 and Myc might involve Gas41. Another protein interacting with Gas41 is the FERM domain-containing protein 4A. FRMD4A also interacts with Yaf9 and has a hypothetical function in cytoskeletal dynamics. The FERM domain is involved in protein-protein interactions and is present in several proteins, primarily involved in structural functions, but also in signaling (kinases and phosphatases) and, interestingly, in Merlin, the neurofibromastosis 2 tumour suppressor protein (Gusella et al., 1999).

We also demonstrate here that Gas41 N-terminal half can substitute in yeast for the corresponding Yaf9 portion and suppress sensitivity phenotypes associated with transcriptional regulation and genetic instability observed in yaf9s strains (Le Masson et al., 2003; Bianchi et al., 2001; 2004). The N-terminal half of these proteins is conserved and contains the YEATS domain which is likely to have conserved functions in different biological systems. The YEATS domain of ENL protein binds histones $\mathrm{H} 3$ and H1 (Zeisig et al., 2005) and is involved in targeting chromatin-modifying complexes (Schultze et al., 2009). The Cterminal half of Gas41, which does not substitute for the C-terminal of Yaf9 in phenotypic suppression and contains a coil-coiled domain required for its incorporation in the SCRAP and Tip60 complexes (Park \& Roeder 2006), might have diverged during evolution, adapting to different protein-protein interactions with coevolving partners.

In conclusion, we have demonstrated that the evolutionarily conserved protein Gas41 can interact with proteins and/or complexes involved in transcription regulation by mechanisms that might be different from those proposed until now, suggesting a multivalent role of this protein in gene expression regulation. We have also found a common regulation of Gas41 and Myc in brain tumour-derived cell lines and biopsies. Finally, the demonstration of functional homology of Gas41 and Yaf9 $\mathrm{N}$-terminal regions confirms that the use of yeast may help to identify the role of Gas41 in brain tumours.

\section{Acknowledgements}

This work was supported by PBZ-MIN-015/ P05/2004 and PRIN 200973ST5Y 002.

\section{REFERENCES}

Amente S, Lania L, Avvedimento EV, Majello B (2010) DNA oxidation drives Myc mediated transcription. Cell Cycle 9: 3002-3004.

Bianchi MM, Costanzo G, Chelstowska A, Grabowska D, Mazzoni C, Piccinni E, Cavalli A, Ciceroni F, Rytka J, Slonimski PP, Frontali L, Negri R (2004) The bromodomain-containing protein Bdf1p acts as a phenotypic and transcriptional multicopy suppressor of YAF9 deletion in yeast. Mol Microbiol. 53: 953-968.

Bianchi MM, Ngo S, Vandenbol M, Sartori G, Morlupi A, Ricci C, Stefani S, Morlino G, Hilger F, Carignani G, Slonimski PP, Frontali L (2001) Large-scale phenotypic analysis reveals identical contributions to cell functions of known and unknown yeast genes. Yeast 18: 1397-1412.

Bittner CB, Zeisig DT, Zeisig BB, Slany RK (2004) Direct physical and functional interaction of the NuA4 complex components Yaf9p and Swc4p. Eukaryot Cell 3: 976-983.

Bradford MM (1976) A rapid and sensitive method for the quantitation of microgram quantities of protein utilizing the principle of protein dye binding. Anal Biochem 7: 248-254.

Casagrande V, Del Vescovo V, Militti C, Mangiapelo E, Frontali L Negri R, Bianchi MM (2009) Cesium chloride sensing and signaling in Saccharomyces cerevisiae: an interplay among the HOG and CWI
MAPK pathways and the transcription factor Yaf9. FEMS Yeast Res 9: 400-410.

Debernardi S, Bassini A, Jones LK, Chaplin T, Linder B, de Bruijn DR, Meese E, Young BD (2002) The MLL fusion partner AF10 binds GAS41, a protein that interacts with the human SWI/SNF complex. Blood 99: 275-281.

Del Vescovo V, Casagrande V, Bianchi MM, Piccinni E, Frontali L, Militti C, Fardeau V, Devaux F, Di Sanza C, Presutti C, Negri R (2008) Role of Hog1 and Yaf9 in the transcriptional response of Saccharomyces cerevisiae to cesium chloride. Physiol Genomics 14: 110120.

Doyon Y, Selleck W, Lane WS, Tan S, Cote J (2004) Structural and functional conservation of the NuA4 histone acetyltransferase complex from yeast to humans. Mol Cell Biol 24: 1884-1896.

Doyon Y, Cote J (2004) The highly conserved and multifunctional NuA4 HAT complex. Curr Opin Gene Dev 14: 147-154.

Fischer U, Heckel D, Michel A, Janka M, Hulsebos T, Meese E (1997) Cloning of a novel transcription factor-like gene amplified in human glioma including astrocytoma grade. J Hum Mol Genet 6: 1817-1822.

Fischer U, Meltzer P, Meese E (1996) Twelve amplified and expressed genes localized in a single domain in glioma. Hum Genet 98: 625628.

Frank SR, Taubert S, Fernandez P, Fuchs M, Chan H, Livingstone D, Amati B (2003) Myc recruit the TIP60 histone acetyltransferase complex to chromatin. EMBO Rep 4: 575-579.

Gangisetty O, Lauffart B, Sondarva GV, Chelsea DM, Still IH (2004) The transforming acidic coiled coil proteins interact with nuclear histone acetyltransferases. Oncogene 23: 2559-2563.

Gusella JF, Ramesh V, MacCollin M, Jacoby LB (1999) Merlin: the neurofibromastosis 2 tumor suppressor. Biochim Biophys Acta 1423: M29-M36.

Harborth J, Weber K, Osborn M (2000) Gas41, a highly conserved protein in eukaryotic nuclei, binds to NuMA. J Biol Chem 275: 31979-31985.

Krogan NJ, Keogh MC, Datta N, Sawa C, Ryan OW, Ding H, Haw RA, Pootoolal J, Tong A, Canadien V, Richards DP, Wu X, Emili A, Hughes TR, Buratowski S, Greenblatt JF. (2003) Snf2 family ATPase complex required for recruitment of the histone H2A variant Htz. Mol Cell 12: 1565-1576.

Le Masson I, Yu DY, Jensen K, Chevalier A, Courbeyrette R, Boulard Y, Smith MM, Mann C (2003) Yaf9, a novel NuA4 histone acetyltransferase subunit, is required for the cellular response to spindle stress in yeast. Mol Cell Biol 23: 6086-6102.

Martinato F, Cesaroni M, Amati B, Guccione E (2008) Analysis of Myc-induced histone modifications on target chromatin. Plos One 3: e3650.

McMahon SB, Wood MA, Cole MD (2000) The essential cofactor TRRAP recruits the histone acetyltransferase hGCN5 to c-Myc. Mol Cell Biol 20: 556-562.

Metzer E, Schule R (2007) The expanding world of histone lysine demethylases. Nat Struct Mol Biol 14: 252-254.

Mizuguchi G, Shen X, Landry J,Wu W-H, Sen S, Wu C (2004) ATPdriven exchange of histone H2AZ variant catalyzed by SWR1 chromatin remodeling complex. Science 303: 343-348.

Park JH, Roeder RG (2006) Gas41 is required for P53 tumor suppressor pathway during normal cell proliferation. Mol Cell Biol 26: 4006-4016.

Patel JH, Du Y, Ard PG, Phillips C, Carella B, Chen CJ, Rakowski C, Chatterjee C, Lieberman PM, Lane WS, Blobel GA, McMahon SB (2004) The c-Myc Oncoprotein is a substrate of the acetyltransferases hGCN5/PCAF and Tip60. Mol Cell Biol 24: 10826-10834.

Perillo B, Ombra MN, Bertoni A, Cuozzo C, Sacchetti S, Sasso A, Chiariotti L, Malorni A, Abbondanza C, Avvedimento EV (2008) DNA oxidation as triggered by $\mathrm{H} 3 \mathrm{~K} 9 \mathrm{me} 2$ demethylation drives estrogen-induced gene expression. Science 319: 202-206.

Perini G, Diolaiti D, Porro A, Della Valle G (2005) In vivo transcriptional regulation of $\mathrm{n}$-Myc target genes is controlled by E-box methylation. Proc Natl Acad Sci USA 102: 12117-22.

Schultze JM, Wang AY, Kobor MS (2009) YEATS domain proteins: a diverse family with many links to chromatin modification and transcription. Biochem Cell Biol 87: 65-75.

Schwab M, Ellison J, Brush M, Rosenau W, Varmus HE, Bishop JM (1984) Enhanced expression of the human gene n-myc consequent to amplification of DNA may contribute to malignant progression of neuroblastoma. Proc Natl Acad Sci USA 81: 4940-4944.

Zeisig DT, Bittner CB, Zeisig BB, Garcia-Cuellar MP, Hess JL, Slany RK (2005) The eleven-nineteen-leukemia protein ENL connects nuclear MLL fusion partners with chromatin. Oncogene 24: 5525-5532.

Zhang H, Roberts DN, Cairns BR (2005) Genome-Wide dynamics of Htz1, a histone $\mathrm{H} 2 \mathrm{~A}$ variant that poises repressed/basal promoters for activation through histone loss. Cell 123: 219-231.

Zimmermann K, Ahrens K, Matthes S, Buerstedde JM, Strätling WH, Phivan L (2002) Targeted disruption of the GAS 41 gene encoding a putative transcription factor indicates that GAS41 is essential for cell viability. J Biol Chem 24: 18626-18631. 